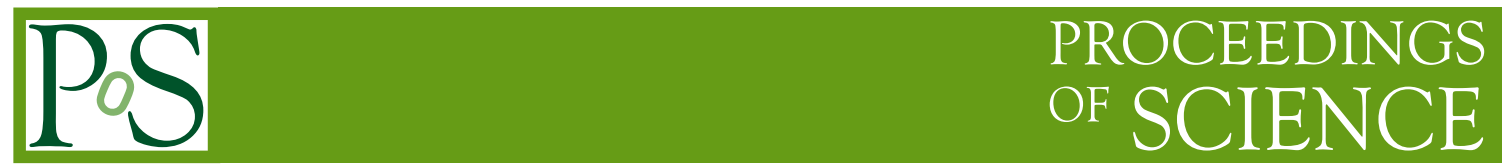

\title{
Lattice Landau gauge quark propagator at finite temperature
}

\section{Paulo J. Silva* and Orlando Oliveira}

CFisUC, Departamento de Física, Universidade de Coimbra, 3004-516 Coimbra, Portugal

E-mail: psilva@uc.pt, orlandodfis.uc.pt

We study the Landau gauge quark propagator, at finite temperature, using quenched lattice simulations. Special focus is given to the behaviour of the momentum space form factors across the confinement-deconfinement phase transition.

XIII Quark Confinement and the Hadron Spectrum - Confinement2018

31 July - 6 August 2018

Maynooth University, Ireland

${ }^{*}$ Speaker. 


\section{Introduction and motivation}

The dynamics of QCD generates dynamically a mass for its fundamental quanta. For the quarks the running mass exceeds by a large amount the contribution due to the Higgs mechanism for small momenta. Indeed, recent lattice simulations at zero temperature of the Landau gauge quark propagator [1,2], taking into account only QCD, show that the running quark mass takes values of $\sim 320-340 \mathrm{MeV}$ at zero momentum for current quark masses below $10 \mathrm{MeV}$. However, in a medium where the fundamental fields feel the effects of temperature and density, as occurs in heavy ion collisions or inside dense stars, the properties observed at zero temperature are changed. At sufficiently high temperature quarks and gluon become deconfined and a chiral phase transition is also expected. For example, lattice simulations within pure SU(3) gauge theories show that the longitudinal component of the Landau gauge gluon propagator at finite temperature is sensitive to the deconfinement phase transition [3]. Moreover, due to the breaking of the center symmetry, the propagators in various $Z_{3}$ sectors differ above $T_{c}$ [4] and the differences can be used to identify the transition to the deconfined regime. The sign problem prevents lattice simulations from covering the full range of realistic chemical potentials $\mu$. The two point correlation functions for the gauge sector for two-color QCD at finite density were investigated in [5] and some low-momentum gluon screening at high densities was observed.

The properties of quarks and, in particular, its propagators change with $T$ and $\mu$. Herein, we report preliminary results for the study of the Landau gauge quark propagator in momentum space, at finite temperature and for temperatures above and below the deconfinement phase transition, using quenched lattice simulations. After gauge fixing, we take into account those configurations that belong to the $Z_{3}$ sector where the phase of the associated Polyakov loop vanishes. There are similar studies where the mass function was measured using Wilson fermions [6], non-perturbative improved Clover fermions [8] and where the quark spectral functions were investigated [7,9] relying on particular ansätze. Our study is performed using much larger lattice volumes and looks also to the bare quark mass dependence of the quark propagator form factors.

\section{Quark Propagator at Finite Temperature}

The simulations with finite temperature break rotational invariance and, therefore, in momentum space the continuum quark propagator is described by three form factors, namely,

$$
\begin{aligned}
S\left(p_{t}, \vec{p}\right) & =\frac{1}{i \gamma_{t} p_{t} \omega\left(p_{t}, \vec{p}\right)+i \vec{\gamma} \cdot \vec{p} Z\left(p_{t}, \vec{p}\right)+\sigma\left(p_{t}, \vec{p}\right)} \\
& =\frac{-i \gamma_{t} p_{t} \omega\left(p_{t}, \vec{p}\right)-i \vec{\gamma} \cdot \vec{p} Z\left(p_{t}, \vec{p}\right)+\sigma\left(p_{t}, \vec{p}\right)}{p_{t}^{2} \omega^{2}\left(p_{t}, \vec{p}\right)+(\vec{p} \cdot \vec{p}) Z^{2}\left(p_{t}, \vec{p}\right)+\sigma^{2}\left(p_{t}, \vec{p}\right)} .
\end{aligned}
$$

In the results reported below, we use non-perturbative improved Clover fermions [10] and consider rotated sources [11]. Furthermore, in the analysis of the lattice data we assume that the simulations are close to the continuum and compute the $\omega\left(p_{t}, \vec{p}\right), Z\left(p_{t}, \vec{p}\right)$ and $\sigma\left(p_{t}, \vec{p}\right)$ form factors relying on Eq. (2.1). On the lattice given that the boundary conditions for fermions are periodic in the spatial directions and anti-periodic in time, the momenta $\vec{p}$ and $p_{t}$ assume discrete values

$$
p_{t}=\frac{2 \pi}{L_{t}}\left(n+\frac{1}{2}\right) \quad \text { and } \quad p_{i}=\frac{2 \pi}{L_{s}} n, \quad \text { where } \quad n=0,1,2, \ldots
$$




\begin{tabular}{cccccc}
\hline $\mathrm{T}(\mathrm{MeV})$ & $\beta$ & $L_{s}^{3} \times L_{t}$ & $\kappa$ & $m_{\text {bare }}(\mathrm{MeV})$ & $c_{s w}$ \\
\hline 243 & 6.0000 & $64^{3} \times 8$ & 0.1350 & 10 & 1.769 \\
& & & 0.1342 & 53 & \\
\hline 260 & 6.0347 & $68^{3} \times 8$ & 0.1351 & 11 & 1.734 \\
& & & 0.1344 & 51 & \\
\hline 275 & 6.0684 & $72^{3} \times 8$ & 0.1352 & 12 & 1.704 \\
& & & 0.1345 & 54 & \\
\hline
\end{tabular}

Table 1: Lattice setup.

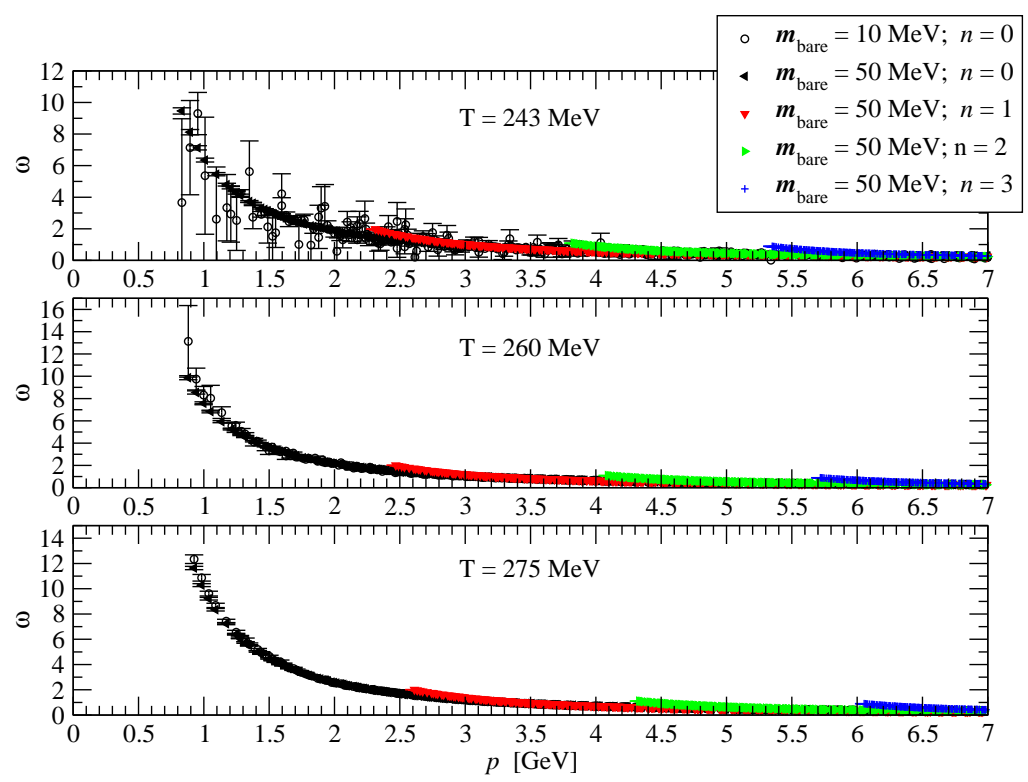

Figure 1: Bare $\omega$, in lattice units, for the various ensembles and for the various Matsubara frequencies.

Further, the "continuum" form factor is given by $Z_{c}\left(p_{t}, \vec{p}\right)=Z\left(p_{t}, \vec{p}\right) / \omega\left(p_{t}, \vec{p}\right)$ and the running quark mass by $M\left(p_{t}, \vec{p}\right)=\sigma\left(p_{t}, \vec{p}\right) / \omega\left(p_{t}, \vec{p}\right)$.

In the current work, these form factors are computed for temperatures around the critical temperature, taken as the critical temperature above which the gluons become deconfined for pure Yang-Mills Theory, i.e. $T_{c} \approx 270 \mathrm{MeV}$. The lattice setup used in the simulations is described in Tab. 1, where each ensemble has one hundred gauge configurations.

The quark propagator is computed inverting the fermionic matrix for 2 point sources. The values used for $c_{s w}$ and the critical hopping parameter $\kappa_{c}$ are computed from [12]. In Tab. 1 the bare quark mass is given by $m_{\text {bare }}=\left(1 / \kappa-1 / \kappa_{c}\right) / 2 a$, where $a$ is given in [3], and our simulations consider $m_{\text {bare }} \approx 10 \mathrm{MeV}$ or $50 \mathrm{MeV}$. 


\section{Results and Conclusions}

In order to suppress lattice effects we have applied momentum cuts as described in [13, 3]. For those quantities defined by ratios of lattice functions, as $Z_{c}$ and $M$, we have ignored all the points whose statistical error was larger than $50 \%$ - this option explains the lack of data points in some of the following plots.

In Fig. 1 we report the bare $\omega\left(p_{t}, \vec{p}\right)$ for all Matsubara frequencies for the heaviest mass considered. For the lightest quark mass only the lowest Matsubara frequency is shown. The plot shows a general trend observed for all simulations: the function associated with the lightest bare quark mass have large fluctuations and, therefore, large statistical errors below the deconfinement phase transition, while above the deconfinement phase transition the statistical fluctuations become similar for both quark masses. Furthermore, at sufficiently high momenta no significative difference between the functions associated to the Matsubara frequencies is seen.

In Fig. 2 the bare $Z_{c}\left(p_{t}, \vec{p}\right)$ is reported. Note that no lattice artefacts have been subtracted. Below $T_{c}$, we observe significant statistical fluctuations associated with the lightest quark mass. Above the deconfinement temperature the lattice data for the lightest quark mass also shows oscillations that deserve further study. The function $Z_{c}\left(p_{t}, \vec{p}\right)$ seems to approach a constant for sufficient large momenta. The not so good agreement between the results for the various Matsubara frequencies at high momenta are a possible indication of the breaking of the $\mathrm{O}(4)$ symmetry, the use of finite lattice spacing and/or finite volume effects.

In Fig. 3 we show the running mass for the various simulations. Note that no removal of the lattice artefacts is performed and that is why the data are shown only up to $p=1.5 \mathrm{GeV}$. For higher momenta the corrections to the use of finite lattice spacing are rather large, making the raw lattice data for the running mass meaningless - see e.g. the discussion and references in [2]. The data shows a $M\left(p_{4}, \vec{p}\right)$ that decreases with the temperature. This effect is illustrated in Fig. 4, where we report $M\left(p_{t}, \vec{p}=0\right)$ for the smallest value of $p_{t}$ for each of the simulations. As we cross the deconfinement temperature, the blue vertical line on the plot, the value of $M$ becomes about half of its values below $T_{c}$. Note, however, that $M$ is always much larger than the bare quark mass, and although the data suggests a decreasing $M(T)$ it can cannot be viewed as an indication of the chiral symmetry restoration.

\section{Acknowledgements}

P.J.S. acknowlegdes the generous sponsorship by the TUM/LMU "Universe Cluster". P.J.S. also acknowledges support by Fundação para a Ciência e a Tecnologia (FCT) under contracts SFRH/BPD/40998/2007 and SFRH/BPD/109971/2015. The authors acknowledge financial support from FCT under contract with reference UID/FIS/04564/2016. The authors also acknowledge the Laboratory for Advanced Computing at University of Coimbra (http://www.uc.pt/lca) for providing access to the HPC resource Navigator. The SU(3) lattice simulations were done using Chroma [14] and PFFT [15] libraries. 


\section{References}

[1] O. Oliveira, A. Kızılersu, P. J. Silva, J. I. Skullerud, A. Sternbeck and A. G. Williams, Acta Phys. Polon. Supp. 9 (2016) 363 doi:10.5506/APhysPolBSupp.9.363 [arXiv:1605.09632 [hep-lat]].

[2] O. Oliveira, P. J. Silva, J. I. Skullerud and A. Sternbeck, arXiv:1809.02541 [hep-lat].

[3] P. J. Silva, O. Oliveira, P. Bicudo and N. Cardoso, Phys. Rev. D 89 (2014) no.7, 074503 doi:10.1103/PhysRevD.89.074503 [arXiv:1310.5629 [hep-lat]].

[4] P. J. Silva and O. Oliveira, Phys. Rev. D 93 (2016) no.11, 114509 doi:10.1103/PhysRevD.93.114509 [arXiv:1601.01594 [hep-lat]].

[5] O. Hajizadeh, T. Boz, A. Maas and J. I. Skullerud, EPJ Web Conf. 175 (2018) 07012 doi:10.1051/epjconf/201817507012 [arXiv:1710.06013 [hep-lat]].

[6] M. Hamada, H. Kouno, A. Nakamura, T. Saito and M. Yahiro, PoS LAT 2006 (2006) 136 doi:10.22323/1.032.0136 [hep-lat/0610010].

[7] F. Karsch and M. Kitazawa, Phys. Rev. D 80 (2009) 056001 doi:10.1103/PhysRevD.80.056001 [arXiv:0906.3941 [hep-lat]].

[8] M. Hamada, H. Kouno, A. Nakamura, T. Saito and M. Yahiro, Phys. Rev. D 81 (2010) 094506. doi:10.1103/PhysRevD.81.094506

[9] O. Kaczmarek, F. Karsch, M. Kitazawa and W. Soldner, Phys. Rev. D 86 (2012) 036006 doi:10.1103/PhysRevD.86.036006 [arXiv:1206.1991 [hep-lat]].

[10] B. Sheikholeslami and R. Wohlert, Nucl. Phys. B 259 (1985) 572. doi:10.1016/0550-3213(85)90002-1

[11] G. Heatlie, G. Martinelli, C. Pittori, G. C. Rossi and C. T. Sachrajda, Nucl. Phys. B 352 (1991) 266. doi:10.1016/0550-3213(91)90137-M

[12] M. Luscher, S. Sint, R. Sommer, P. Weisz and U. Wolff, Nucl. Phys. B 491 (1997) 323 doi:10.1016/S0550-3213(97)00080-1 [hep-lat/9609035].

[13] R. Aouane, V. G. Bornyakov, E. M. Ilgenfritz, V. K. Mitrjushkin, M. Muller-Preussker and A. Sternbeck, Phys. Rev. D 85 (2012) 034501 doi:10.1103/PhysRevD.85.034501 [arXiv:1108.1735 [hep-lat]].

[14] R. G. Edwards, B. Joo, Nucl. Phys. Proc. Suppl. 140, 832 (2005) [arXiv: hep-lat/0409003].

[15] M. Pippig, SIAM J. Sci. Comput. 35, C213 (2013). 

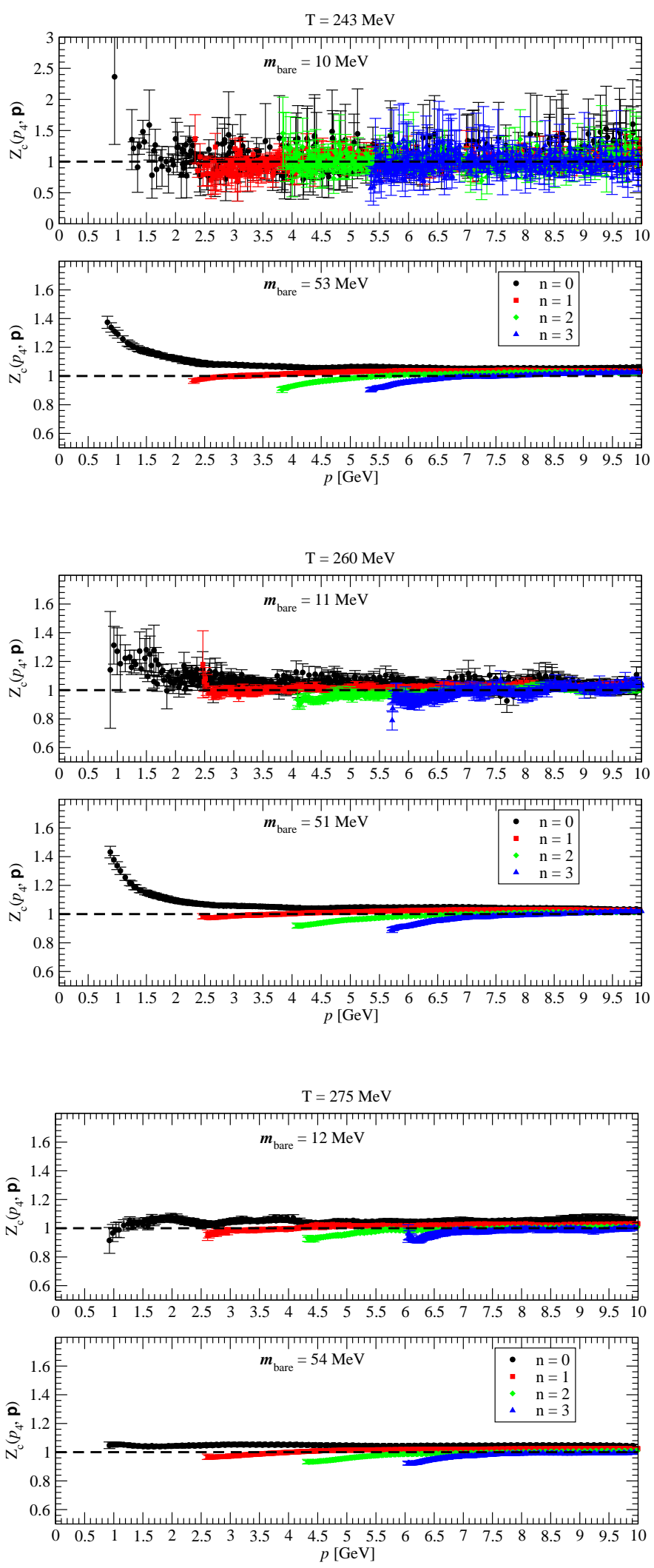

Figure 2: $\operatorname{Bare} Z_{c}\left(p_{t}, \vec{p}\right)$ for the various simulations. 

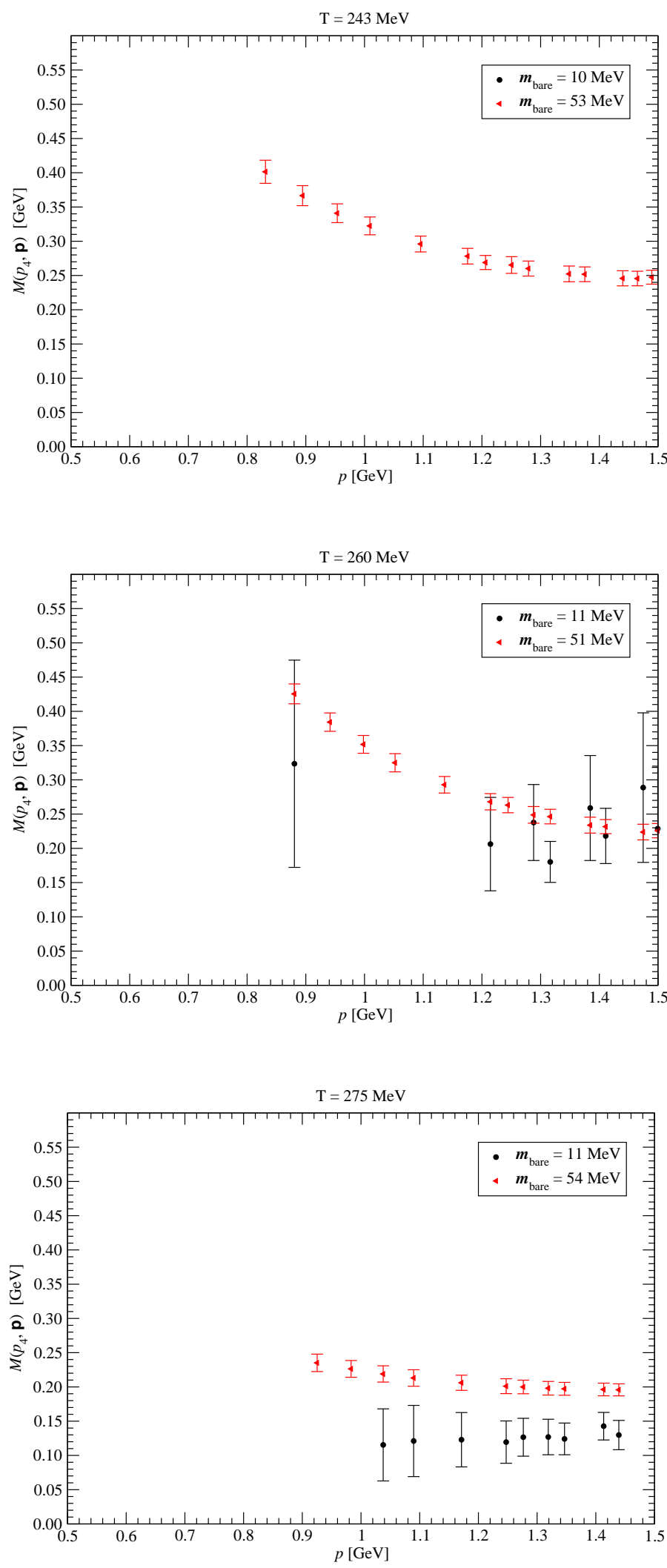

Figure 3: Running quark mass $M\left(p_{t}, \vec{p}\right)$ for the various simulations. Note that we ignored data points with large errors — see text for details. 


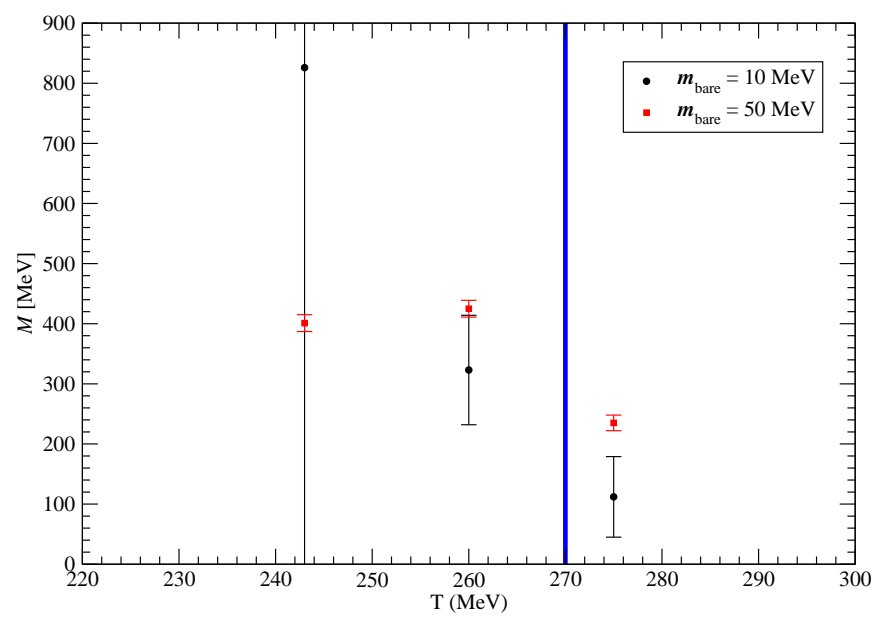

Figure 4: $M\left(p_{t}, \vec{p}=0\right)$ for the smallest $p_{t}$ for the various ensembles. 